\title{
Prevalencia del estado de desnutrición en los adultos mayores de la Unidad Médica Familiar Núm. 53 de León, Guanajuato, México
}

\author{
Prevalence of the state of malnutrition in older adults in Family Medical \\ Unit No. 53 in Leon, Guanajuato, Mexico
}

\author{
Lucía Elizabeth Fuentes-Pimentel, * Alicia Camacho-Guerrero
}

\begin{abstract}
RESUMEN. Introducción: El pobre estado de nutrición de los ancianos es preocupante. La desnutrición contribuye a que decline la salud, pues afecta la función y el estado cognitivo e incrementa el uso de los servicios de salud, la institucionalización prematura y la mortalidad. Objetivo: Conocer la prevalencia de desnutrición en los adultos mayores de la Unidad Médica Familiar (UMF) Núm. 53 de León, Guanajuato. Material y métodos: Estudio transversal, descriptivo y prospectivo, que fue realizado de julio a octubre de 2018 e incluyó derechohabientes mayores de 60 años, adscritos a la UMF Núm. 53 León, Gto; se excluyeron pacientes con deterioro cognitivo y se eliminaron casos con respuestas erróneas. De acuerdo con el MiniNutritional Assessment se consideró: malnutrición < 17 puntos, riesgo de malnutrición de 17 a 23.5 puntos y satisfactorio $\geq 24$ puntos. El análisis de resultados se realizó con estadística descriptiva. Resultados: Se estudiaron 146 adultos mayores, de los cuales el 62\% fue de mujeres y el $38 \%$ de hombres. La edad promedio fue de 69.24 años. Se encontró malnutrición en el $25.34 \%$, riesgo de malnutrición en el $49 \%$ y normal en el $26 \%$. En tanto a la malnutrición se refiere, estuvieron desnutridos el $24.9 \%$ de ellos, con sobrepeso el $27.02 \%$ y obesidad el $48.64 \%$. Conclusiones: La prevalencia de malnutrición es mayor que lo encontrado en la literatura nacional (11.3\%). Los médicos familiares debemos evaluar el estado nutricional en esta población al implementar medidas terapéuticas para mejorar su calidad de vida.
\end{abstract}

Palabras clave: Malnutrición, mini-encuesta nutricional, adulto mayor.

ABSTRACT. Introduction: The poor state of nutrition in the elderly population is an area of concern. Malnutrition contribute to the decline of health, affecting function and cognitive status, increasing the use of health services, premature institutionalization and mortality. Objective: To know the Prevalence of Malnutrition in Older Adults of the UMF No. 53 of Leon, Gto. Material and methods: Transversal, descriptive, prospective study. That took place from July to October 2018, where right holders over 60 years,

* Residente del tercer año de la Especialidad en Medicina Familiar. Unidad de Medicina Familiar (UMF) Núm. 53, Instituto Mexicano del Seguro Social (IMSS), México.

₹ Especialista en Medicina Familiar. UMF Núm. 53, IMSS. Coordinadora Auxiliar Médica de Educación en Salud, Delegación IMSS, León, Gto México.

Recibido: 29 de Octubre de 2019. Aceptado: 27 de Febrero de 2020.
Correspondencia:

Dra. Lucía Elizabeth Fuentes-Pimentel

Fernando Montes de Oca Núm. 166

Col. Chapultepec Norte Morelia, CP 58260 ,

Michoacán, México.

E-mail: lucylu_liz@hotmail.com

Conflicto de intereses: Todos los autores declaran que no existe ningún conflicto de intereses con respecto a la publicación de este artículo. 
attached to UMF No. 53 Leon, Gto.; Patients with cognitive impairment were excluded and cases with erroneous responses were eliminated. According to the Mini Nutritional Assessment: malnutrition: <17 points, risk of malnutrition: 17 to 23.5 points and satisfactory nutritional status $\geq 24$ points. The analysis of results is performed with descriptive statistics. Results: 146 older adults were studied, women 62\% and 38\% men. The average age was 69.24 years. The distribution of nutritional status was malnutrition in $25.34 \%$, risk of malnutrition in $49 \%$ and normal in $26 \%$. Of the patients with malnutrition, $24.9 \%$ were malnourished, $27.02 \%$ overweight and 48.64\% obese. Conclusions: The prevalence of malnutrition is higher than that found in national literature $(11.3 \%)$, which requires that as family physicians we evaluate the nutritional status in this population to implement therapeutic measures with the objective of improving their quality of life.

Keywords: Malnutrition, mini-nutritional survey, older adults.

\section{INTRODUCCIÓN}

El envejecimiento de la población mundial avanza cada año en una proporción del $2 \%$ en los adultos mayores de 60 años. ${ }^{1}$ Se calcula que en el planeta viven 600 millones de personas mayores de 60 años, aunque se proyecta que para el año 2025 se duplicará esta población y para 2050 ascenderá a dos mil millones de personas. $^{1,2}$

En México, en 1970, la distribución de edad de la población tenía forma piramidal, es decir, una base amplia y una cúspide angosta, pues el $50 \%$ de la población era menor de 15 años. En el año 2000 se observó una pirámide abultada en el centro, lo que refleja un aumento de las personas en edades medias y una disminución de la proporción de los menores de cinco años. ${ }^{3}$ Se prevé que en el año 2050 haya una mayor proporción de población en senectud, debido a la disminución de las tasas de natalidad y al aumento de la esperanza de vida. Si bien el envejecimiento de la población se presenta en todo el mundo, la diferencia entre países radica en la planeación y preparación para afrontar este cambio. $\mathrm{El}$ incremento de la esperanza de vida es un indicador de mejoría del estado de salud de la población en edades tempranas, pero en edades mayores se relaciona con tasas elevadas de enfermedades crónicas y degenerativas e incapacidad, las cuales afectan su calidad de vida. ${ }^{4-6}$ Las enfermedades registradas con más frecuencia fueron las siguientes: hipertensión arterial, problemas articulares, reumatismo, desnutrición y osteoporosis. ${ }^{6}$ México cuenta con poca información del perfil de salud y nutrición del adulto mayor y de las características de su entorno.

\section{Evaluación del estado de nutrición del adulto mayor}

Se define como el ejercicio clínico realizado por el médico en el que se realizará una evaluación y una medición de variables nutricionales, las cuales pueden ser de diversos tipos, como: clínicas, antropométricas, bioquímicas y dietéticas. Esta evaluación se realiza de una manera integral con el fin de proporcionar información sobre el estado de nutrición en el que se encuentra el adulto mayor. Podemos llevar a cabo todo lo anterior en diferentes escenarios, por ejemplo: en la consulta externa, hospitalización, terapia intensiva, evaluación preoperatoria y otros, teniendo como objetivo final tener un plan de manejo nutricional.

Desnutrición. Se puede definir como el resultado de una ingestión alimenticia que se realiza de forma continuada, pero que no es suficiente para cubrir las necesidades de energía alimentaria por absorción y/o uso biológico deficiente de los nutrientes consumidos, ${ }^{7-9}$ teniendo en cuenta que el $20 \%$ del peso habitual se ha perdido en por lo menos seis meses o menos y que puede ocurrir una grave disfunción fisiológica. ${ }^{10}$

El diagnóstico de desnutrición en el adulto mayor se establece con la presencia de dos o más de los siguientes criterios: pérdida de peso $\geq 5 \%$ en un mes o $\geq 10 \%$ en seis meses respecto al peso habitual.

El Índice de Masa Corporal (IMC) se establece con los siguientes criterios: $<21 \mathrm{~kg} / \mathrm{m}^{2}$, al- 
búmina en sangre $<3.5 \mathrm{~g} / \mathrm{dL}$, puntaje del MiniNutritional Assessment (MNA) < 17 y circunferencia de pantorrilla $<31 \mathrm{~cm} .{ }^{11}$

Malnutrición. Podemos definirla como un estado en el que existen carencias, excesos o desequilibrios en la ingestión de energía, proteínas y/o algunos otros nutrientes que ocasionan efectos adversos en el tejido corporal, en la composición o en la función que tiene consecuencias clínicas. ${ }^{9,10}$

Obesidad. La Organización Mundial de la Salud (OMS) la define como: «el exceso en la grasa corporal hasta un punto en que es nocivo para la salud; utiliza los mismos puntos de corte con base en el IMC para definir el sobrepeso y la obesidad en adultos». De esta forma, los cambios en la composición corporal podrían subestimar la obesidad basada en el IMC, por lo que en este grupo de edad se considera un IMC de 23 a 27.49 $\mathrm{kg} / \mathrm{m}^{2}$ como normal. Una circunferencia de cadera mayor de $90 \mathrm{~cm}$ en hombres y $80 \mathrm{~cm}$ en mujeres se considera obesidad abdominal. ${ }^{12}$

Obesidad sarcopénica. Aparece cuando existe pérdida de masa muscular en combinación con aumento de la masa grasa. Se define como la coexistencia de sarcopenia y el porcentaje de grasa corporal mayor correspondiente a un IMC de $27 \mathrm{~kg} / \mathrm{m}^{2}$ (> 27\% de grasa corporal en hombres y $>38 \%$ de grasa corporal en mujeres). ${ }^{10}$

\section{Mini-Nutritional Assessment (MNA)}

La evaluación mínima del estado de nutrición en pacientes geriátricos (MNA) es un instrumento estandarizado y validado que permite obtener una evaluación rápida del estado nutricional de los individuos de la tercera edad y evaluar su riesgo de desnutrición, con el fin de proporcionar una intervención nutricional oportuna y sin la necesidad de un equipo especializado de evaluación nutricional. ${ }^{13,14}$ Esta prueba consta de 18 ítems divididos en cuatro categorías: parámetros antropométricos, estado general del paciente, encuesta dietética y valoración subjetiva; clasifica a las personas mayores, sobre un máximo de 30 puntos, en tres grupos: estado nutricional satisfactorio (S) ( $\geq 24$ puntos), riesgo de malnutrición (RM) (17-23.5 puntos) y malnutrición $(\mathrm{M})(<17$ puntos $) .{ }^{15}$

\section{Evaluación del nivel socioeconómico (NSE AMAI 2018) ${ }^{16}$}

El nivel socioeconómico (también llamado estatus socioeconómico) es una medida total económica y sociológica que combina la preparación laboral de una persona y la posición económica y social individual o familiar, las cuales se basan en los ingresos, en la educación y en el empleo. Las variables que se toman en cuenta en esta herramienta son: nivel educativo del jefe del hogar, número de baños completos en la vivienda, número de autos en el hogar, conexión a internet en el hogar, número de integrantes en el hogar mayores de 14 años que trabajan y número de dormitorios. Debido a los cambios físicos que el paciente geriátrico presenta, este sector de la población se vuelve muy vulnerable a distintas enfermedades; por esto, como médicos familiares debemos evitar o disminuir la gravedad de las afecciones comunes en este sector de la población.

Con este preámbulo, se ha desarrollado esta investigación con el fin de garantizar una vejez más digna para los adultos mayores pertenecientes al Instituto Mexicano del Seguro Social mediante la valoración y análisis de su estado nutricional, para que se diseñe un modelo efectivo que mejore su condición de vida y su estado nutricional. Por esta razón, y ya que es un problema de salud pública, nos hemos propuesto el siguiente objetivo de investigación: conocer la prevalencia de la desnutrición en los adultos mayores de la UMF Núm. 53 de León, Guanajuato (México).

\section{MATERIAL Y MÉTODOS}

Se realizó un estudio transversal, descriptivo, prospectivo y observacional, el cual se llevó a cabo de julio a octubre de 2018 y se incluyeron derechohabientes mayores de 60 años que estuvieran adscritos a la UMF 53 de León, Gto. Se excluyeron pacientes con deterioro cogni- 
tivo y se eliminaron los casos con respuestas erróneas.

De acuerdo con el Mini-Nutritional Assessment, se consideró como malnutrición a los individuos que obtuvieron menos de 17 puntos, como riesgo de malnutrición a aquéllos que obtuvieron de 17 a 23.5 puntos y como estado nutricional satisfactorio a aquéllos con 24 puntos o más. Utilizamos estadística descriptiva; la expresión de las características categóricas se realizó con frecuencia simple y porcentajes. Las variables discretas se expresaron con media \pm desviación estándar. Para el análisis de resultados se utilizó el programa de computación SPSS 21. El cálculo del tamaño de muestra se realizó con la fórmula para diferencia de proporciones.

\section{RESULTADOS}

Se incluyeron 146 adultos mayores pertenecientes a la UMF Núm. 53 en la ciudad de León, Guanajuato. Éstos se distribuyeron en 90 (62\%) mujeres y 56 (38\%) hombres. Su edad promedio fue de 69.24 años \pm 7.67 años (máxima de 89 años y mínima de 60 años).

El índice de masa corporal de los adultos mayores fue de $23.83 \pm 2.61$. En la Figura 1 se muestra el estado de nutrición de acuerdo con el índice de masa corporal (IMC). Se presentó un estado de desnutrición moderada en el 10.95\%, desnutrición leve en el $10.25 \%$ y un estado nu-

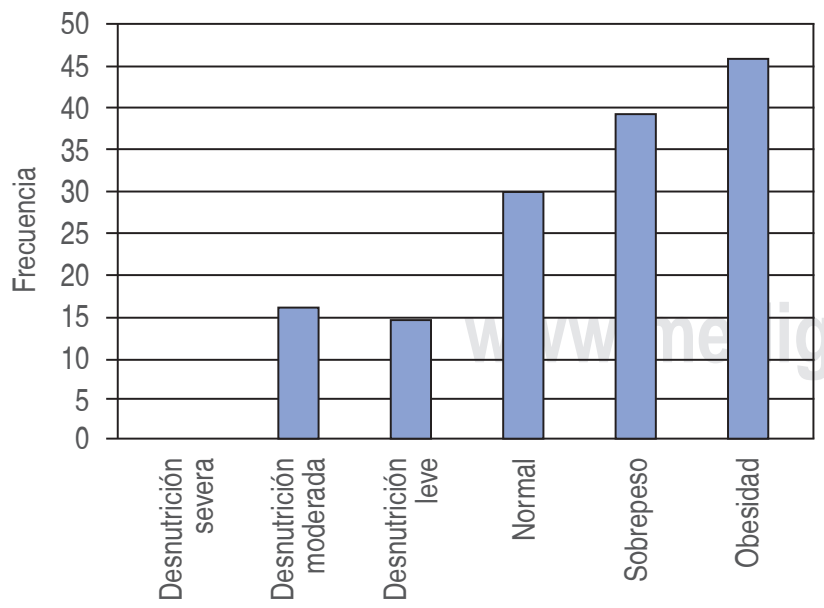

Figura 1: Estado de nutrición por índice de masa corporal.

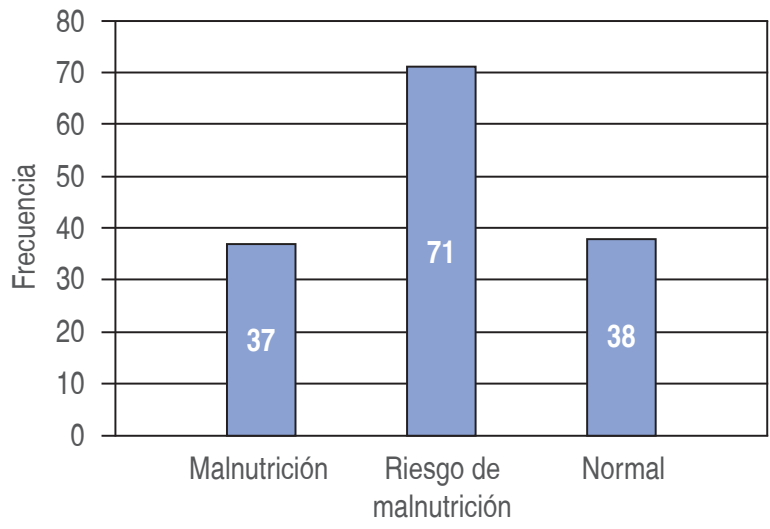

Figura 2: Nivel de desnutrición.

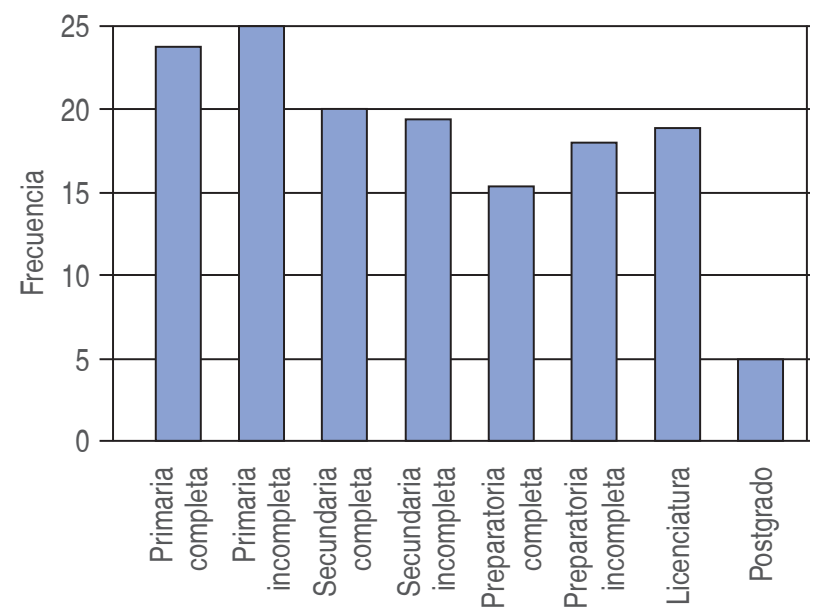

Figura 3: Nivel de escolaridad.

tricional satisfactorio o normal en el $20.5 \%$, así como sobrepeso en el $26.71 \%$ y obesidad en el $31.50 \%$; no se encontró un estado de desnutrición severa.

La Figura 2 muestra la clasificación del nivel de desnutrición con base en el Mini-Nutritional Assessment. De esta manera el $25.34 \%$ presentó malnutrición, el 49\% riesgo de malnutrición y el $26 \%$ se encontró dentro de lo normal.

En la Figura 3 se muestra el grado de escolaridad de los adultos mayores; predomina la primaria incompleta en el $17.12 \%$ y primaria completa en el $16.43 \%$; el $13.69 \%$ respondió que su grado escolar fue secundaria completa y el 13.01\% secundaria incompleta; el 10.95\% respondió que tenía la preparatoria completa, mientras que el $12.32 \%$ no terminó la prepara- 
toria; asimismo el $13.01 \%$ cursó la licenciatura y el 3.04\% realizó estudios de postgrado.

La Figura 4 muestra la clasificación del nivel socieconómico de acuerdo con el NSE AMAI 2018 , clasificándose el $2.05 \%$ como clase alta, el $8.2 \%$ como clase media-alta, el $11.64 \%$ como clase media, el $21.91 \%$ como media-baja, el $23.97 \%$ como baja-alta y el $32.19 \%$ como baja-baja. La distribución de malnutrición y riesgo de malnutrición de acuerdo con la edad, el sexo y la escolaridad se presenta en las Tablas 1 a 3.

En la Tabla 4 se observa que, en la distribución del estado de nutrición de acuerdo con el nivel socieconómico en la clase alta hubo un mayor porcentaje de pacientes con riesgo de malnutrición; en la clase media-alta predominó el estado de malnutrición, mientras que en la media y media-baja el mayor porcentaje de pacientes se encontró con riesgo de malnutrición; en la clase baja-alta y baja-baja el riesgo de malnutrición fue igualmente mayor.

En la Tabla 5 se observa que, en la clase alta, media-alta y media predominaron los pa-

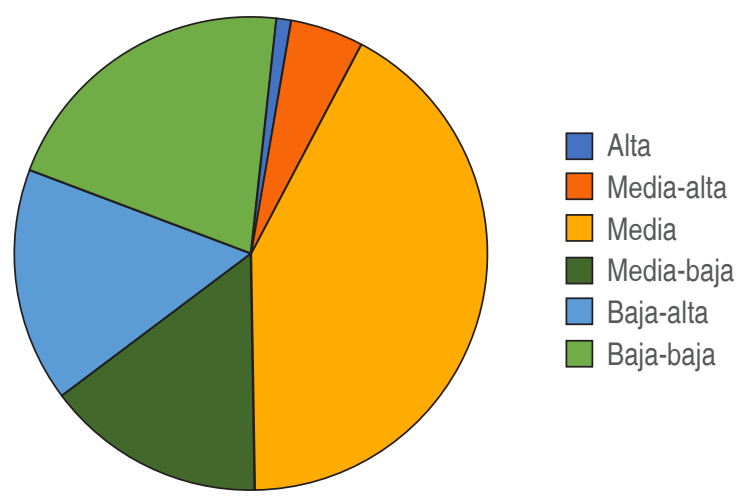

Figura 4: Nivel socioeconómico.

\begin{tabular}{|cccc|}
\hline \multicolumn{4}{c|}{ Tabla 1: Distribución de malnutrición por edad. } \\
\hline Edad & $\begin{array}{c}\text { Malnutrición } \\
n(\%)\end{array}$ & $\begin{array}{c}\text { Riesgo } \\
\text { malnutrición } n(\%)\end{array}$ & $\begin{array}{c}\text { Normal } \\
n(\%)\end{array}$ \\
\hline $49-59$ & 0 & $1(100.0)$ & 0 \\
$60-69$ & $22(26.19)$ & $41(48.80)$ & $21(25.00)$ \\
$70-79$ & $11(23.90)$ & $25(54.34)$ & $10(21.73)$ \\
$>80$ & $4(26.60)$ & $4(26.60)$ & $7(46.60)$ \\
\hline
\end{tabular}

\begin{tabular}{|c|c|c|c|}
\hline Sexo & $\begin{array}{c}\text { Malnutrición } \\
n(\%)\end{array}$ & $\begin{array}{c}\text { Riesgo } \\
\text { malnutrición } \\
\mathrm{n}(\%)\end{array}$ & $\begin{array}{l}\text { Normal } \\
\mathrm{n}(\%)\end{array}$ \\
\hline Masculino & $16(17.7)$ & 28 (31.1) & $12(13.30)$ \\
\hline Femenino & 21 (23.3) & $43(47.7)$ & $26(28.80)$ \\
\hline
\end{tabular}

Tabla 3: Distribución de malnutrición por escolaridad.

\begin{tabular}{cccc} 
Escolaridad & $\begin{array}{c}\text { Malnutrición } \\
\mathrm{n}(\%)\end{array}$ & $\begin{array}{c}\text { Riesgo } \\
\text { malnutrición } \\
\mathrm{n}(\%)\end{array}$ & $\begin{array}{c}\text { Normal } \\
\mathrm{n}(\%)\end{array}$ \\
\hline $\begin{array}{c}\text { Primaria } \\
\text { completa }\end{array}$ & $8(33.3)$ & $8(33.30)$ & $8(33.30)$ \\
incompleta & $9(36.0)$ & $9(36.00)$ & $7(28.00)$ \\
Secundaria & & & \\
completa & $5(25.0)$ & $11(55.00)$ & $4(20.00)$ \\
incompleta & $7(36.8)$ & $9(47.36)$ & $3(15.78)$ \\
Preparatoria & & & \\
completa & $4(25.0)$ & $7(43.75)$ & $5(31.25)$ \\
incompleta & $2(11.1)$ & $12(66.6)$ & $4(22.20)$ \\
Licenciatura & $1(5.2)$ & $13(68.42)$ & $5(26.31)$ \\
Postgrado & $1(20.0)$ & $2(40.00)$ & $2(40.00)$ \\
\end{tabular}

cientes con obesidad; no así en la media-baja en la que hubo una mayor prevalencia de pacientes con sobrepeso; en la clase baja-alta fue igual el porcentaje de quienes se encuentran en sobrepeso con los que se encuentran con desnutrición. Por último, en la clase baja-baja el mayor porcentaje fue de los pacientes que padecen obesidad y sobrepeso. En la Tabla 6 se muestra la distribución del estado de nutrición por IMC en los pacientes con malnutrición; en éstos predominaron aquéllos con obesidad.

\section{DISCUSIÓN}

En este estudio se presenta un análisis sobre el estado de malnutrición en los adultos mayores de la Unidad de Medicina Familiar Núm. 53 de León, Guanajuato. Actualmente, la malnutrición es un problema de salud pública en auge 
en nuestro país, el cual afecta sobre todo a las personas de mayor edad y pasa desapercibida ante los profesionales de la salud. Los adultos mayores están sujetos a numerosos riesgos nutricios, lo que tiene un efecto directo en su calidad de vida.

En el presente estudio se encontraron a los adultos mayores con prevalencia elevada de estado de malnutrición y de riesgo de malnutrición al compararlo con los resultados del estudio realizado en la Ciudad de México por Vanessa Mota Sanhua y colaboradores, ${ }^{17}$ quienes encontraron una prevalencia de malnutrición de $11.3 \%$ y una prevalencia de riesgo de malnutrición de 59\%; sin embargo, otro estudio en España encontró cifras mayores que las de nuestro estudio, con prevalencia de malnutrición de 47.6\% ${ }^{18}$ Con base en el sexo, el mayor predominio correspondió a las mujeres. La preponderancia femenina tiene una relación de 3:2

\begin{tabular}{|c|c|c|c|}
\hline \multicolumn{4}{|c|}{$\begin{array}{l}\text { Tabla 4: Distribución del estado de nutrición } \\
\text { por nivel socioeconómico. }\end{array}$} \\
\hline $\begin{array}{l}\text { Nivel } \\
\text { socioeconómico }\end{array}$ & $\begin{array}{l}\text { Malnutrición } \\
\mathrm{n}(\%)\end{array}$ & $\begin{array}{c}\text { Riesgo } \\
\text { malnutrición } \\
\mathrm{n}(\%)\end{array}$ & $\begin{array}{l}\text { Normal } \\
\mathrm{n}(\%)\end{array}$ \\
\hline Alta $(\mathrm{A} / \mathrm{B})$ & $1(33.30)$ & $2(66.60)$ & 0 \\
\hline Media-alta (C+) & $4(33.30)$ & $5(41.60)$ & $3(25.00)$ \\
\hline Media $\left(C_{+}\right)$ & $3(17.65)$ & 10 (58.82) & $4(23.52)$ \\
\hline Media-baja (C) & $10(31.25)$ & $16(50.00)$ & $6(18.75)$ \\
\hline Baja-alta (D+) & $10(28.57)$ & 18 (51.42) & $7(20.00)$ \\
\hline Baja-baja $(E)$ & $9(19.14)$ & $20(42.55)$ & 18 (38.29) \\
\hline
\end{tabular}

sobre los hombres en desnutrición, lo cual es consistente con los hallazgos de otros trabajos. ${ }^{19}$

A lo largo de la vida, el género implica distintos riesgos y desventajas con respecto a la salud; los padecimientos crónicos tienen mayor frecuencia e impacto en las mujeres de la tercera edad, puesto que en ellas se detectan las prevalencias más altas, las cuales están asociadas normalmente con otros padecimientos. Además, las mujeres en este país enfrentan desventajas educacionales, económicas y sociales, lo que las hace más vulnerables dentro del grupo de ancianos.

El porcentaje más alto de malnutrición se presentó en los grupos con primaria incompleta, completa y secundaria incompleta, lo cual nos indica que el nivel de alfabetización sí podría llegar a ser un determinante en el riesgo de malnutrición en el adulto mayor, porque hay desconocimiento de una adecuada alimentación y de la pirámide nutricional; además refleja las consecuencias de consumir alimentos industrializados y/o altos en carbohidratos, las repercusiones del sedentarismo, el tabaquismo, el alcoholismo y la importancia de tener acceso a la información sobre los servicios de salud, vacunas, medidas preventivas, etc.

El nivel socioeconómico está relacionado con el nivel de nutrición; en el presente estudio encontramos que el mayor porcentaje de pacientes con desnutrición se encontró en el estrato socioeconómico de clase baja-alta y, por el contrario, el mayor porcentaje de obesidad se encontró en el estrato socioeconómico de clase alta y media-alta. Esto fue comparable con los

Tabla 5: Distribución del estado nutricional por índice de masa corporal según el nivel socioeconómico.

$\begin{array}{lcccc}\text { Nivel socioeconómico } & \text { Obesidad } n(\%) & \text { Sobrepeso } n(\%) & \text { Normal } n(\%) & \text { Desnutrición } n(\%) \\ \text { Alta (A/B) } & 2(66.60) & 1(33.30) & 0 & 0 \\ \text { Media-alta (C+) } & 7(58.33) & 3(25.00) & 1(8.30) & 1(8.30) \\ \text { Media (C+) } & 8(47.05) & 5(29.41) & 4(23.50) & 0 \\ \text { Media-baja (C) } & 9(28.12) & 12(37.50) & 5(15.62) & 6(18.75) \\ \text { Baja-alta (D+) } & 9(25.71) & 11(31.42) & 4(11.42) & 11(31.42) \\ \text { Baja-baja (E) } & 15(31.91) & 15(31.91) & 8(17.02) & 9(19.14)\end{array}$


Tabla 6: Distribución del estado de nutrición por índice de masa corporal en malnutrición.

\begin{tabular}{lccc}
$\begin{array}{l}\text { Nivel } \\
\text { desnutrición }\end{array}$ & $\begin{array}{c}\text { Desnutrición } \\
\mathrm{n}(\%)\end{array}$ & $\begin{array}{c}\text { Sobrepeso } \\
\mathrm{n}(\%)\end{array}$ & $\begin{array}{c}\text { Obesidad } \\
\mathrm{n}(\%)\end{array}$ \\
\hline Malnutrición & $9(24.32)$ & $10(27.02)$ & $18(48.64)$
\end{tabular}

resultados de estudios anteriores en los que se encontró también mayor prevalencia de obesidad y sobrepeso, sobre todo en las clases alta y media. ${ }^{20}$ Ello se debe a que en el estrato socioeconómico bajo no se llega a alcanzar la cantidad de nutrimentos necesarios para alcanzar un adecuado aporte calórico-proteico, lo cual está condicionado por un insuficiente ingreso económico; mientras que, por el contrario, en el estrato socioeconómico alto sí hay un ingreso económico suficiente para cubrir los grupos básicos alimenticios que son necesarios para una adecuada nutrición; sin embargo, se observa también un exceso en la ingesta de azúcares y carbohidratos, lo que explica así el sobrepeso y obesidad presentado en estos pacientes.

Entre las limitaciones del estudio se puede señalar que el mayor predomino del sexo femenino ocurrió porque la selección de adultos mayores se realizó a través de un muestreo no probabilístico de casos consecutivos, lo que predispone a reclutar en mayor número a la población que demanda con mayor frecuencia los servicios de atención médica, como es el caso de las mujeres.

En México, los adultos mayores poseen un estado de salud y nutrición inadecuado, lo cual es urgente atender para optimizar su calidad de vida. Los determinantes de la desnutrición son múltiples y diversos y, por tanto, para estable- cer si un adulto mayor presenta o no desnutrición se debe evaluar de forma intencionada la situación física, nutricional, económica y social del adulto mayor, además de las mediciones antropométricas tradicionales.

Partiendo de los resultados del estudio, se concluye que es necesario o imprescindible diseñar estrategias para mejorar el estado nutricional de nuestros adultos mayores, así como también promoverlas en los profesionales de la salud, con el fin de lograr una detección oportuna que disminuya las comorbilidades para que se otorgue una atención de calidad y calidez a los derechohabientes, al favorecer su estado de salud.

\section{Aspectos éticos}

El presente estudio se apegó a lo dispuesto en el Reglamento de la Ley General de Salud en materia de investigación para la salud, Título Segundo, Capítulo I, artículo 13 y 14, Fracción I, V, VI, VII Y VIII se ajustó a los principios éticos y científicos que lo justificaron. Este estudio se consideró de riesgo mínimo para los participantes. Para que el consentimiento informado fuera considerado existente, el sujeto de investigación recibió una explicación clara y completa, según el artículo 21, fracciones I, II, III, IV, V, VI, VII, VIII y X. De acuerdo a la Declaración de Helsinki de la Asociación Médica Mundial: Principios Éticos para la investigación Médicas Apartado I: Fracción V, VII, VIII. Apartado II: Fracción I, II. Apartado V, VI, VII Fracción I, II. Sometido al comité local de investigación de la Unidad de Medicina Familiar No 53, Delegación Estatal en Guanajuato del Instituto Mexicano del Seguro Social y autorizado con el número de registro: R-2018-1008-010.

\section{BIBLIOGRAFÍA}

1. Organización de las Naciones Unidas. Segunda Asamblea Mundial Sobre el Envejecimiento [Internet]. Nueva York: 2002. Disponible en: https://undocs.org/ es/A/CONF.197/9

2. United Nations. Department of Economic and Social Affairs, Population Division. World population ageing 1950-2050. New York: UN; 2002.
3. Zúñiga E, Vega D. Envejecimiento de la población de México, reto del siglo XXI. México: Consejo Nacional de Población; 2004.

4. Shamah-Levy Teresa, Cuevas-Nasu Lucía, Mundo-Rosas Verónica, Morales-Ruán Carmen, Cervantes-Turrubiates Leticia, Villalpando-Hernández Salvador. Estado de salud y nutrición de los adultos mayores en México: resultados 
de una encuesta probabilística nacional. Salud Pública Mex [revista en la Internet]. 2008 [citado el 5 de agosto de 2017]; 50 (5): 383-389. Disponible en: http://www. scielo.org. $\mathrm{mx} /$ scielo.php? script=sci_arttext\&pid=S003636342008000500011\&lng=es.

5. Peláez M, Pratts O, Hennis AJ, Ham-Chande R, León Díaz EM, Lebrão ML et al. Encuesta de Salud, Bienestar y Envejecimiento (SABE); metodología de la encuesta y perfil de la población estudiada. Rev Panam Salud Pública. 2005; 17 (5-6): 307-322.

6. Secretaría de Salud. Programa de Acción: Atención al Envejecimiento. México: Secretaría de Salud, Subsecretaría de Prevención y Protección de la Salud; 2001.

7. Castro V, Gómez-Dantés H, Negrete-Sánchez J, TapiaConyer R. Las enfermedades crónicas en las personas de 60-69 años. Salud Publica Mex. 1996; 38: 438-447.

8. Olaiz G, Rojas R, Barquera S, Shamah T, Aguilar C, Cravioto P et al. Encuesta Nacional de Salud. 2000. Tomo 2. La salud de los adultos. Cuernavaca, México: Instituto Nacional de Salud Pública; 2003.

9. De la Mata, C. Malnutrición, desnutrición y sobrealimentación. Revista Médica Rosario. 2008; 74: 17 20. Disponible en: http://www.bvsde.ops-oms.org/texcom/ nutricion/mata.pdf.

10. García-Zenón T, Villalobos-Silva JA. Malnutrición en el anciano. Parte 1: desnutrición, el viejo enemigo. Medicina Interna de México. 2012; 28 (1): 57-64. Disponible en: http:// cmim.org/boletin/pdf2012/MedIntContenido01_09.pdf.

11. Raynaud-Simon A, Revel-Delhom C, Hébuterne X; French Nutrition and Health Program, French Health High Authority. Clinical practice guidelines from the french health high authority: nutritional support strategy in protein-energy malnutrition in the elderly. Clin Nutr. 2011; 30 (3): 312-319. doi: 10.1016/j.clnu.2010.12.003. Epub 2011 Jan 19. PMID: 21251732.
12. Alberti KG, Zimmet P, Shaw J, IDF Epidemiology Task Force Consensus Group. The metabolic syndrome: a new worldwide definition. Lancet. 2005; 24-30: 30-366: 1059-1062. Disponible en: https://www.ncbi.nlm.nih.gov/ pubmed/16182882.

13. Velásquez AM. Desnutrición en los adultos mayores: La importancia de su evaluación y apoyo nutricional. Respyn. 2011; 12 (2): 105.

14. Söderhamn U, Dale B, Sundsli K, Söderhamn O. Nutritional screening of older home dwelling norwegians: a comparison between two instruments. Clin Interv Aging. 2012; 7: 383-391.

15. Jiménez SM, Sola VJ, Pérez RC, Turienzo LM, Larrañaga LG, Mancebo SM et al. Estudio del estado nutricional de los ancianos de Cantabria. Nutr Hosp. 2011; 26 (2): 345-354.

16. Asociación Mexicana de Agencias de Inteligencia de Mercado y Opinión [Internet]. Comité de Nivel Socioeconómico AMAI, Noviembre de 2017. [Acceso 27 de febrero del 2020] Disponible en: http://www.amai.org/nse/ wp-content/uploads/2018/04/Nota-Metodolo\%CC\%81gicoNSE-2018-v3.pdf.

17. Mota SV, Levin PG, Rivas AL. Estado de Nutrición de Adultos Mayores de un sector marginado en la Ciudad de México. An Med (Mex). 2012; 57 (3): 205-209.

18. Vaca BR, Ancizu GI, Moya GD. Prevalencia de desnutrición en personas mayores institucionalizadas en España: un análisis multicéntrico nacional. Nutr Hosp. 2015; 31 (3): 1205-1216.

19. Sánchez H, Leyva J. Prevalencia de Malnutrición en adultos Mayores. Unidad de Medicina Familiar No. 51, León, Gto, México. Octubre, 2011, p. 28.

20. Cárdenas-Quintana H, Roldan AL. Relación entre el estado nutricional y el nivel socioeconómico de adultos mayores no institucionalizados. Rev Chil Nutr. 2013; 40 (4): 343-350. 